\title{
COSMOLOGICAL SIMULATIONS WITH SMOOTHED PARTICLE HYDRODYNAMICS
}

\author{
Mario G. Abadi , Diego G. Lambas and Patricia B. Tissera \\ IATE-Observatorio Astronomico Cordoba, Argentina. \\ CONICET, Argentina.
}

We have developed and tested a code that computes the evolution of a mixed system of gas and dark matter in expanding world models. The gravitational forces are calculated with the Adaptative P3M algorithms developed by $\mathrm{H}$. Couchmann, 1993. The calculation of gas forces follow the standard SPH formalism (Monaghan, 1989).

The system expands according to a cosmological solution and consist of a cube with periodic boundary conditions. Our simulations run typically with 5000 gas particles and 32000 collisionless particles.

We have tested the code using the Lazer-Irvine equation (cosmic energy conservation) modified to include the presence of gas particles.

We have also analyzed the ability of the code to reproduce the self-similar solution for the acretion to a spherically symmetric perturbation in a homogeneous expanding background of a collisional gas. Figure 1 show the dimensionless density (D), pressure (P), mass $(M)$ and velocity $(V)$ profiles vs Lambda (radius/turn-around radius) and the corresponding analytical solutions found by Bertschinger, 1985. The vertical bar in the figures indicates the shock radius.

The inclusion of energy dissipation allows the segregation of the dark and the baryonic components and the formation of disk systems. We follow the colapse of a rotating spherical pertubation of 512 gas particles with initial dimensionless spin parameter 0.04 . In figure $2 \mathrm{a}, \mathrm{b}$ are shown the final particle distributions for the gas projected into the $\mathrm{xy}$ and $\mathrm{xz}$ plane respectively.

Future research will be centered in several cosmological applications of this code which will take into account star formation.

We have benefited with useful discussions with Dr. Simon White and Dr. Julio Navarro.

This work has been supported by grants from CONICET, CONICOR and The British Council, Argentina. 

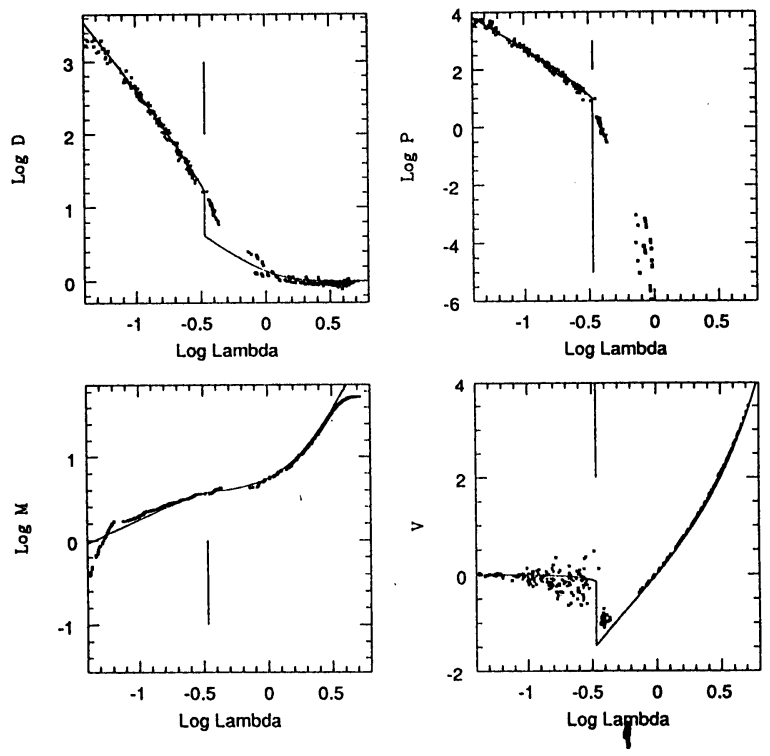

Figure 1

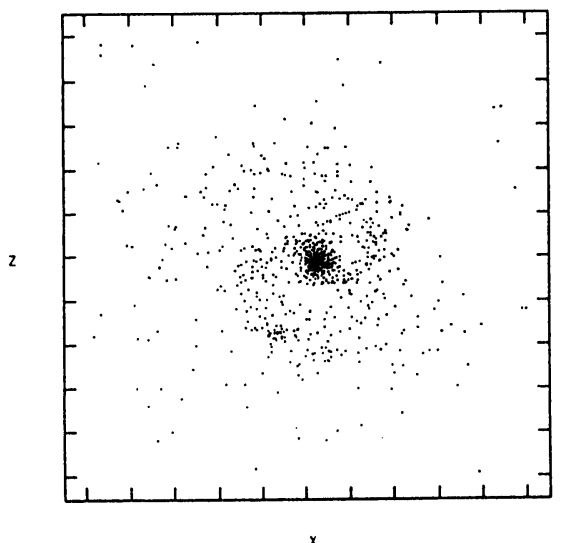

Figure 20

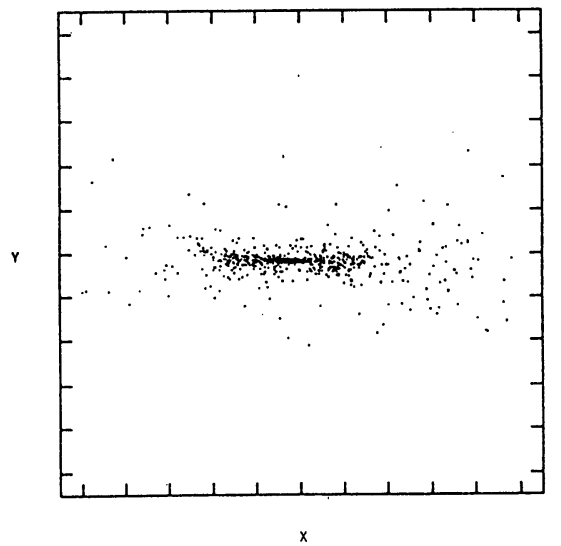

Figure 2b 